\title{
Bidimensional chronoabsorptometric study of electropolymerisation of 4,4'-bis(2-methylbutylthio)-2,2'-bithiophene
}

\author{
Virginia Ruiz a , Álvaro Colina a , Aránzazu Heras ${ }^{a}$, Jesús López-Palacios a,*, \\ Renato Seeber ${ }^{b}$ \\ a Área de Química Analítica, Facultad de Ciencias, Universidad de Burgos, E-09001 Burgos, Spain \\ b Dipartimento di Chimica, Università di Modena e Reggio Emilia, I-41100 Modena, Italy
}

Received 6 February 2002; received in revised form 28 February 2002; accepted 1 March 2002

\begin{abstract}
Bidimensional chronoabsorptometry is a novel spectroelectrochemical technique that monitors simultaneously three different signals: current and absorbance both normal to the electrode plane and parallel to this plane during a time in which a fixed potential is imposed. This technique is applied in the visible range to the study of the electropolymerisation of 4,4'-bis(2-methylbutylthio)2,2'-bithiophene (MBTBT). Experiments are performed in a spectroelectrochemical cell under finite diffusion conditions (thin layer cell) with the aim of interpreting the processes taking place both at the electrode surface and in the adjacent solution during the potentiostatic electrogeneration and deposition of the polymer. Correlations are drawn out among the trends of the oligomers concentration in solution, the polymer electrodeposition and charging, and the current flow, on the time scales of the different steps of the process. (C) 2002 Elsevier Science B.V. All rights reserved.
\end{abstract}

Keywords: Bidimensional chronoabsorptometry; Conducting polymers; Electropolymerisation; Polythiophenes; Spectroelectrochemistry

\section{Introduction}

Conducting polymers, in particular polythiophenes, have attracted large attention in the last two decades thanks to the many possible different applications [1-4]. Several properties of these materials are the object of intensive research devoted to develop novel applications, to study the mechanisms of the charge transport in the bulk of the polymer, as well as at the polymer|solution interface [5-7], and to tailor the sought characteristics by modification of the chemical structure and of the synthesis conditions [8-11]. A wide number of analytical techniques have been used with the aim of collecting information on the electrochemical and connected properties of this class of materials. In particular, electrochemical techniques have been applied to characterise newly developed macromolecular compounds with respect to the relevant charge-discharge processes [12-14]. Furthermore, several studies are reported about

\footnotetext{
${ }^{*}$ Corresponding author. Fax: +34-947-25-88-31.

E-mail address: jlopal@ubu.es (J. López-Palacios).
}

the growth mechanism of thiophene-based polymer films on the basis of purely electrochemical data, coming both from potentiostatic [15] and from potentiodynamic measurements [16-20].

On the other hand, since one of the most interesting characteristics of this type of materials lies in the electrochromic properties, spectroscopic techniques have proved to constitute most suitable tools for complementing the data from electrochemical measurements. Thus, a number of spectroscopies have been used for different purposes. In particular, in situ spectroelectrochemical measurements in the UV-visible region have provided for information on the redox switching mechanism of polythiophenes [7,21-25], on the polymerisation and deposition processes [26,27], on the nature of the species present in solution during the electropolymerisation [28], and on the characteristics of polythiophene films [29]. In situ external reflectance FTIR spectroscopy has also provided for an insight into the polymerisation mechanism and into the $\mathrm{p}$ - and n-doping mechanisms of different substituted polythiophenes [30,31]. Since p-doping causes significant 
changes in the vibrational and electronic properties of the polymer, Raman spectroscopy has recently been employed as a sensitive tool in similar studies [25,32]. Absorption and fluorescence spectroscopy, in their turn, have been used to obtain information on the conformation of several oligomers and polymers based on thiophene chains [33].

Electrochemical and spectroelectrochemical results have also been complemented by electrochemical quartz crystal microbalance (EQCM) measurements, in order to parallel the previous cited data with those of the mass changes occurring during both electrodeposition [14] and doping and de-doping processes [7,30,34]. Additional techniques, like electron paramagnetic resonance (EPR) [32], in situ conductivity [7,18,24,31,34], and electrochemical impedance spectroscopy (EIS) [35] have been employed both to investigate the $\mathrm{p}$ - and $\mathrm{n}$-doping and corresponding de-doping processes and to characterise the electrode|polymer|solution system.

Many literature reports deal with the influence of the electrochemical polymerisation conditions on the characteristics of the resulting polymers, paying attention at the electrical, optical, and mechanical properties, as well as at the stability under repetitive potential cycling. Apparently contradictory results obtained by different researchers [9] clearly indicate that the experimental conditions play a very critical role in the electrochemical synthesis. The reactivity of the starting compound, which is considerably influenced by steric and electronic effects of the substituents [9], constitutes a first important factor affecting the characteristics of the resulting polymer. The use of bithiophene instead of thiophene derivatives as the starting compounds is preferable thanks to the lower oxidation potential [36], that allows many polymers to be obtained under relatively mild conditions, thus avoiding possible overoxidation. In particular, $\beta, \beta^{\prime}$-disubstituted bithiophenes bearing electron-donor substituents, such as thioalkyl groups, have proved to be easily electrogenerated, leading to quite stable polymers $[12,13,18]$.

The design of monomer structures suitable to achieve a definite increase of conductivity has not been satisfactorily successful so far. Further progress on this direction requires a better understanding of the factors governing the electropolymerisation. As a matter of fact, several intermediate steps are not fully elucidated, which is a necessary condition to generation of conducting polymers with most suitable conformation. The difficulty of obtaining a trustworthy interpretation of the electropolymerisation process on the basis of the results from a single, though powerful technique has been underlined previously [27,37]. Whenever several techniques have been used at the same time on the same system, much more information has been collected $[27,37]$. In agreement with this point of view, bidimensional chronoabsorptometry has been employed in the present work. Bidimensional spectroelectrochemistry is a novel and powerful technique that has been applied successfully to the study of chemical systems of interest in many different fields [38]. In particular, this approach has proved to be a suitable one in the analysis of processes leading to deposition onto electrodes, such as the electropolymerisation of different 4,4'-bis(alkylthio)- 2,2'-bithiophenes: namely, in the potentiodynamic electrosynthesis of poly $\left[4,4^{\prime}-\right.$ bis(metylthio)-2,2'-bithiophene] [38] and of poly[4,4'-bis(butylthio)-2,2'-bithiophene] [39]. It is possible to monitor simultaneously one electrochemical and two spectroscopic signals. A former one on the direction perpendicular to the electrode, looking at the whole chemistry involved in the electrochemical reaction, i.e., both at the final products, either deposited on the electrode or remaining in the solution, and at intermediate species in the solution, and a latter one on the direction parallel to the electrode surface, only looking at species in the solution. In this way, simultaneous in situ monitoring of the whole absorbance accounted for by electrodeposited polymer and species in solution $\left(A_{\mathrm{N}}\right.$ : normalbeam arrangement) and that only due to species in solution ( $A_{\mathrm{P}}$ : parallel-beam arrangement), along with the electrochemical signal, are possible throughout the electropolymerisation. Actually, in order to obtain the absorbance only due to the deposited polymer, i.e., $A_{\mathrm{N}}^{\mathrm{C}}$, the absorbance in normal-beam arrangement, $A_{\mathrm{N}}$, has to be corrected by subtracting the value of the weighted parallel-beam absorbance at the same wavelength. Since the parallel path length is longer than the normal one, $A_{\mathrm{P}}$ has to be weighted by the factor $\omega / l$ (optical path lengths ratio) [38]. In Eq. (1)

$A_{\mathrm{N}}^{\mathrm{C}}=A_{\mathrm{N}}-A_{\mathrm{P}} \times \frac{\omega}{l}$

$\omega$ is the thickness of the diffusion space (optical path length in the normal direction) and $l$ is the electrode width (optical path length in the parallel direction). Properly weighted $A_{\mathrm{P}}$ accounts for the contribution of the soluble species to the absorbance measured along the normal direction. This correction leads to exact results only when the concentration profile of the absorbent species in solution is almost plane or when the measured parallel absorbance takes small values. If a great gradient in the concentration profile is present, the proposed correction becomes only a rough approximation, better in any case than overlooking the contribution to $A_{\mathrm{N}}$ of species in solution. The big advantage offered by the bidimensional approach lies in the fact that it gives joint but independent information about the species involved in every step of the electropolymerisation, since it looks distinctly at different products and, therefore, it allows an analysis of how they are singly affected by changes in the experimental conditions. 


\section{Experimental}

\subsection{Chemicals}

Acetonitrile (AN) was used as solvent and tetrabutylammonium hexafluorophosphate $\left(\mathrm{TBAPF}_{6}\right)$ as supporting electrolyte. All chemicals were of analytical grade and used without further purification.

4,4'-bis(metylbutylthio)-2,2'-bithiophene (MBTBT) was kindly supplied by Schenetti, Department of Chemistry, University of Modena and Reggio Emilia.

\subsection{Instrumentation}

Chronoabsorptometric experiments were carried out in a conventional three-electrode system controlled by an EG\&G-PAR Versastat II (Perkin Elmer, USA) potentiostat. A light source DH-2000 (Top Sensor Systems, The Netherlands) with double lamp, i.e., halogen and deuterium, and a modular system composed of two spectrometers and two diode array detectors with 2048 elements (SD2000 Ocean Optics, USA) were assembled using optic fibres, as previously described [38].

The spectroelectrochemical cell, designed and built in our laboratory, has been reported elsewhere [38]. An optically transparent gold film sputtered over a glass slide was used as the working electrode, a platinum wire as the auxiliary electrode, and a $\mathrm{Ag} / \mathrm{AgCl} / \mathrm{KCl}$, $3 \mathrm{~mol} \mathrm{dm}^{-3}$ system, assembled in a micropipette plastic tip, as the reference electrode. The diffusion layer thickness is limited to $150 \mu \mathrm{m}$ and the parallel-beam samples the whole width and length $(3 \mathrm{~mm})$ of the layer where the soluble species are confined during electropolymerisation.

Throughout all the experiments, absorbance was measured taking as reference (zero) the value of the absorbance of the starting solution (dimer + supporting electrolyte). The dimer does not absorb in the spectral range under the experimental conditions (concentration and optical path length).

\section{Results and discussion}

Previous researches $[28,39]$ have shown that a number of intermediate species of different nature are present in solution during the potentiodynamic polymerisation of thiophenes: both neutral and cationic species have been detected during cyclic potential sweeps. In the present paper absorptometric measurements, recorded in parallel arrangement during both potentiodynamic and potentiostatic polymerisation of MBTBT, have also evidenced the formation of soluble chromophores under different experimental conditions. In the potentiodynamic polymerisation the $A_{\mathrm{P}}$ absorbance was found to vary at varying the potential according to quite different behaviours, depending on the selected wavelength. As an example, voltabsorptometric curves relative to the first potential cycle between the limits 0.00 to $+1.10 \mathrm{~V}$, recorded on an MBTBT, AN solution, at two extreme wavelength values characteristic of neutral and positively oxidised species, i.e., at 400 and $700 \mathrm{~nm}$, respectively, have been normalised and reported in Fig. 1. $A_{\mathrm{P}}$ measured at $700 \mathrm{~nm}$ is strongly dependent on the electrode potential: it increases progressively in the anodic scan, also increases past the switching potential, and reaches a maximum value in the first portion of the reverse scan. Accordingly, the cationic species are generated at positive potentials, being then reduced in the backward sweep, as proved by the decrease that follows the absorbance maximum. On the other hand, $A_{\mathrm{P}}$ at 400 $\mathrm{nm}$ accounts for neutral species in solution generated by radical-radical coupling of oxidised dimer with subsequent (fast) release of two protons. These species continue to diffuse into the bulk solution once the potential scan is reversed until attaining a maximum concentration whose value remains unchanged throughout the backward sweep. The absence of any increase during the backward potential scan is in agreement with the high rate of the radical-radical coupling reaction. The decrease of the absorbance due to positively charged species agrees with the hypothesised electrode reduction as a cause of the decrease of concentration of charged oligomeric species, i.e., of $A_{\mathrm{P}}$ at $700 \mathrm{~nm}$. In the subsequent cycles the trend of the absorbance exhibits a similar behaviour, the overall $A_{\mathrm{P}}$ increasing from one cycle to the following one.

The results collected under potentiodynamic conditions (voltabsorptometry) are however of difficult interpretation, due to the complexity and variability of the whole scheme of the processes occurring at the different applied potentials. This suggested carrying out the oxidative electropolymerisation at a constant potential,

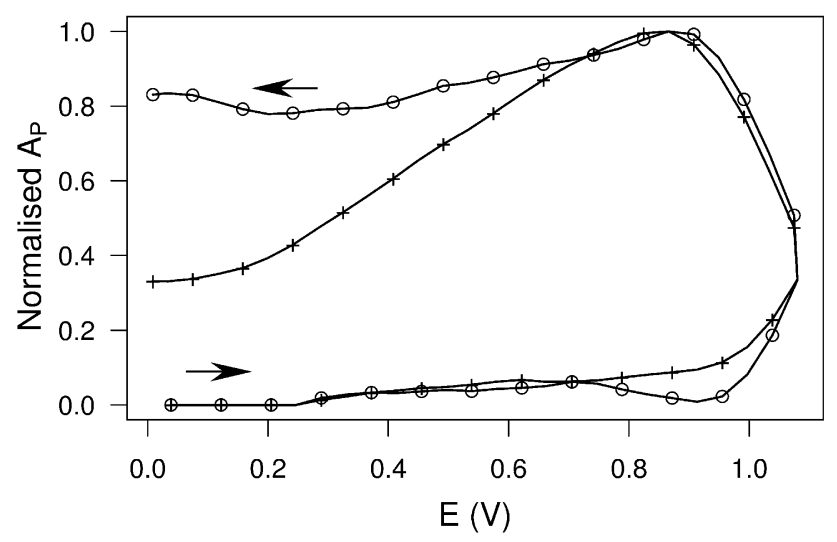

Fig. 1. Voltabsorptograms at $400(\circ)$ and $700 \mathrm{~nm}(+)$, corresponding to the first cycle of a series of 10 subsequent cyclic potential sweeps between 0.00 and $+1.10 \mathrm{~V}$ at scan rate $0.01 \mathrm{~V} \mathrm{~s}^{-1} .5 \times 10^{-3} \mathrm{~mol} \mathrm{dm}^{-3}$ MBTBT, $0.1 \mathrm{~mol} \mathrm{dm}^{-3} \mathrm{TBAPF}_{6}$, AN solution. 
thus simplifying the operative conditions, the extent of occurrence of the different processes only depending on the concentrations of the species, rather than on the variable potential.

Electropolymerisation of MBTBT under potentiostatic conditions (chronoabsorptometry) was accomplished using various dimer concentrations, polarising the electrode at different positive potentials, for different time length. As already evidenced, the experiments were carried out under finite diffusion conditions. Absorption spectra in the visible range, i.e., from 350 to $800 \mathrm{~nm}$, were recorded in both arrangements, simultaneously with current. As an example, Fig. 2 shows the 3D plots of absorbance in normal (Fig. 2(a)) and parallel (Fig. 2(b)) arrangement vs. wavelength and electropolymerisation time, recorded during a $300 \mathrm{~s}$ chronoabsorptometric experiment at $+1.12 \mathrm{~V}$. Spectra recorded at the same time in the two arrangements are different from each other, which confirms our initial assumption that different species are monitored in the two configurations: $A_{\mathrm{N}}^{\mathrm{C}}$ computed according to Eq. (1) accounts for the deposited polymer on the electrode surface, while the intermediate oligomeric species generated in solution are accounted for by $A_{\mathrm{P}}$. As a first observation, it should be
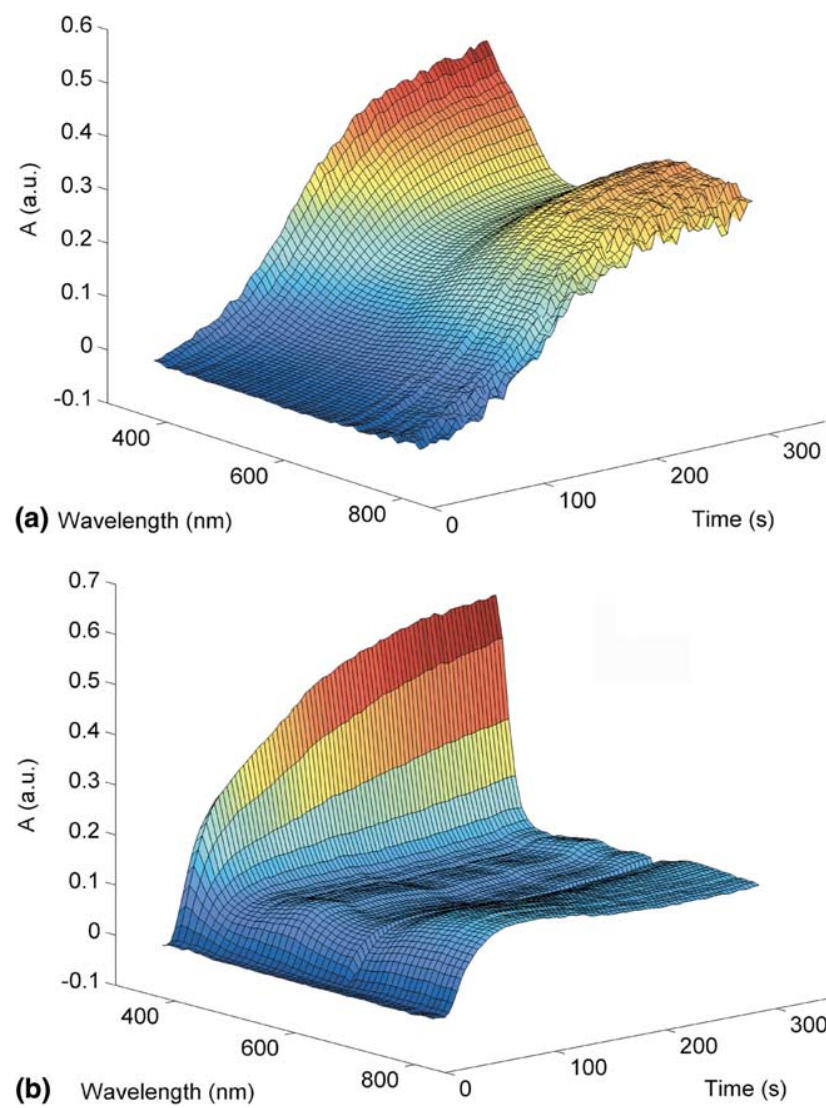

Fig. 2. 3D plots of absorbance in normal (a) and parallel (b) arrangement vs. wavelength and time for a $300 \mathrm{~s}$ chronoabsorptometric experiment at +1.12 V. $5 \times 10^{-3} \mathrm{~mol} \mathrm{dm}^{-3}$ MBTBT, $0.1 \mathrm{~mol} \mathrm{dm}^{-3}$ $\mathrm{TBAPF}_{6}, \mathrm{AN}$ solution. noted that $A_{\mathrm{N}}^{\mathrm{C}}$ increases during the whole experiment, in accordance with the increase of the polymer thickness on the electrode. This also implies that the MBTBT in the diffusion space is not exhaustively consumed during the chronoamperometric test. On the other hand, $A_{\mathrm{P}}$ varies differently with time depending on the selected wavelength. The same wavelengths used in potentiodynamic experiments, i.e., 400 and $700 \mathrm{~nm}$, have been chosen: the trends of the relevant $A_{\mathrm{N}}^{\mathrm{C}}$ and $A_{\mathrm{P}}$ value at time passing are reported in Figs. 3(a) and (b), respectively. The electrical charge spent has also been plotted in the same figures. To convenient comparison, each one of the three signals has been normalised by dividing by its maximum value.

The absorbance values at $400 \mathrm{~nm}$ (Fig. 3(a)) exhibit a very different behaviour depending on the optical path direction. In the normal arrangement, where electrodeposited species absorb, the sigmoidal-shaped $A_{\mathrm{N}}^{\mathrm{C}}$ vs. time curve displays the steepest quasi-linear increase at intermediate times. Conversely, the steepest and major increase of $A_{\mathrm{P}}$, which accounts for neutral soluble, shortchain oligomers, occurs in the correspondence of the first times of electropolymerisation, rising at a slower rate afterwards. Therefore, most of the electrical charge involved at the beginning of the experiment is spent in generating short-chain oligomeric intermediates. Only once concentration and length of the oligomers are high enough, the absorbance due to the deposited polymer starts rising sharply. In this stage, polymer growth takes place on a polymer-coated electrode: a quasi-linear dependence of absorbance on time is observed, the slope decreasing markedly when reaching a final step limited by the dimer supply.

As regards the absorbance at $700 \mathrm{~nm}($ Fig. $3(\mathrm{~b})), A_{\mathrm{N}}^{\mathrm{C}}$ values exhibit similar trends: once the contribution of the oxidised oligomers in solution is subtracted to $A_{\mathrm{N}}$, the absorbance at any wavelength only accounts for the amount of oxidised polymer on the electrode surface, whose time-dependence has just been discussed. Quite nicely, $A_{\mathrm{P}}$, which accounts for cationic oligomeric intermediates in solution, shows a maximum coincident with the starting point of the quasi-linear increase of $A_{\mathrm{N}}^{\mathrm{C}}$. At longer times, the concentration of such cationic oligomers is practically unchanged in the diffusion layer, justifying the previous observation that the polymer on the electrode continues growing.

Since the derivative of the absorbance exhibits, for a simple system, a shape similar to that of the current, possessing however the advantage of being selective, that is to say it is only sensitive to processes in which absorbing species are involved, plots of the derivative of the absorbance with respect to time are commonly employed in voltabsorptometric studies. On the contrary, plotting time-derivative absorbance is not common practice in chronoabsorptometric experiments, even though the observed trends can actually provide for quite interesting 


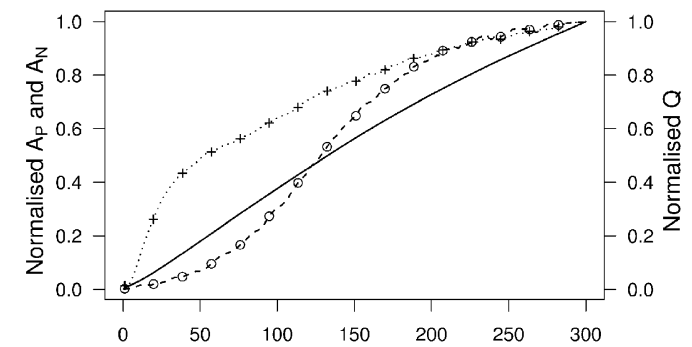

(a)

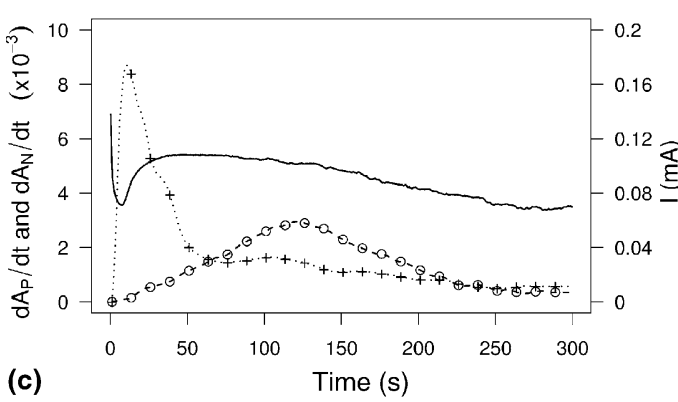

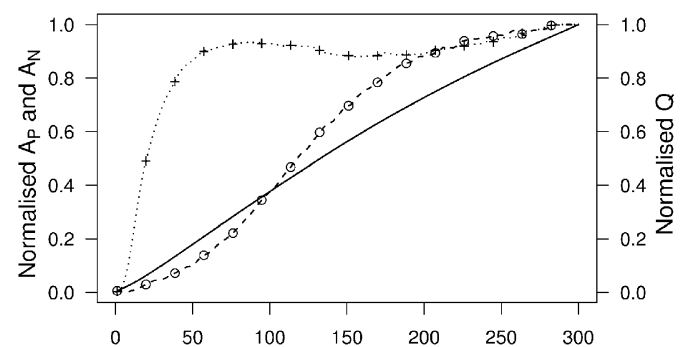

(b)

Time (s)

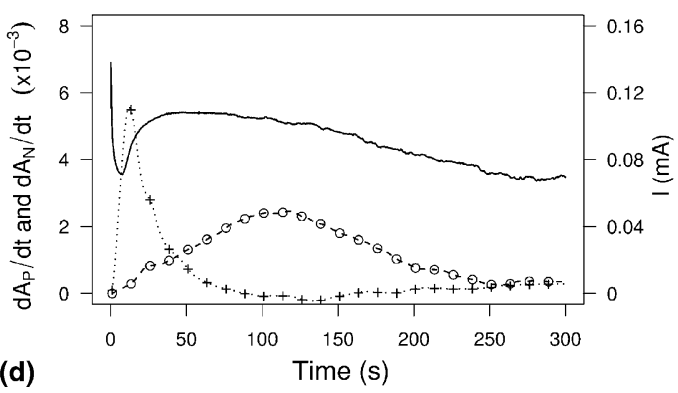

Fig. 3. Normalised $A_{\mathrm{N}}^{\mathrm{C}}(\circ), A_{\mathrm{P}}(+)$ absorbances at $400 \mathrm{~nm}$ (a) and $700 \mathrm{~nm}$ (b), with corresponding electrical charge collected (continuous line), plotted vs. time. Corresponding derivative chronoabsorptograms at $400 \mathrm{~nm}$ (c) and $700 \mathrm{~nm}$ (d), with relevant current intensity, plotted vs. time. Experimental conditions as in Fig. 2.

information. However, neither normal nor parallel spectral measurements are separately capable to complete the electrochemical information: bidimensional spectroelectrochemical measurements become in such a situation an invaluable tool, as the following results emphasise. The derivative of the absorbance with respect to time in both normal and parallel configurations, i.e., $\mathrm{d} A_{\mathrm{N}} / \mathrm{d} t$ and $\mathrm{d} A_{\mathrm{P}} / \mathrm{d} t$, together with the current flowing through the electrode, are plotted vs. time in Figs. 3(c) and (d), at $\lambda=400$ and $700 \mathrm{~nm}$, respectively.

The maximum of the $\mathrm{d} A_{\mathrm{P}} / \mathrm{d} t$ vs. time curves is located at $t \approx 10 \mathrm{~s}$, which should identify the period in which the highest rate for generation of both neutral and charged short-chain oligomers is reached. This maximum is almost coincident with the location of the minimum in the $i / t$ curve, which means that the charge spent until that time is only devoted to form oligomers in solution, as confirmed by the low values of the derivative and original (Fig. 3(a)) chronoabsorptograms in normal arrangement. However, after reaching a maximum value, the oligomers generation rate drops sharply, while the current intensity increases as a result of both the considerable increase of the electroactive area due to nucleation process and the beginning of the p-doping process of the polymer, which has started depositing onto the electrode [13]. Accordingly, a subsequent, continuous growth of $\mathrm{d} A_{\mathrm{N}} / \mathrm{d} t$ parallels the drop of $\mathrm{d} A_{\mathrm{P}} / \mathrm{d} t$, until reaching a maximum in the polymer generation rate that corresponds to the quasi-linear portion in Fig. 3(a). From that point onwards, the current intensity gradually decreases along with the generation rate of neutral oligomers and, consequently, the rate of polymer growth lowers as well. This notwithstanding, as previously noticed, the dimer in the diffusion space is not totally consumed throughout the chronoamperometric test.

As occurs at $400 \mathrm{~nm}$, the maximum in the $\mathrm{d} A_{\mathrm{P}} / \mathrm{d} t$ curve and the minimum in the chronoamperometric current are time-coincident at $700 \mathrm{~nm}$ (see Fig. 3(d)); however, after reaching a maximum, the generation rate of cationic oligomers decreases less sharply than the generation rate of neutral short oligomers does: a significant concentration of charged oligomeric species is steadily present in the solution, as the relevant plot in Fig. 3(b) clearly shows.

By analysing the bidimensional spectroscopic signals, the explanation for the trend in the $i / t$ chronoamperometric curve [13] can find sound support. At very short times, the current has to be for the most part ascribed to the diffusion-controlled oxidation of the starting dimer. In a second time interval, a minimum current value is reached in correspondence with the highest oligomerisation rate. Subsequently, the chain propagation takes place via the ascertained $(\mathrm{CE})_{n}$ [or $\left.(\mathrm{CCE})_{n}\right]$ mechanism, i.e., via radical-radical coupling, proton release, and subsequent electrochemical oxidation steps, as far as the critical chain length is reached and polymer starts to precipitate onto the electrode (nucleation); finally, the deposited polymer undergoes p-doping, that is to say, it is oxidised. Of course, all the preceding steps occur in parallel to the last ones to a different extent. During the last stage, once a layer of polymer covers the surface, the current becomes nearly constant, due to progressive formation of charged polymer multilayers (growing), 
following the same radical-radical coupling mechanism as in the nucleation step. When the process has been carried out in an even thinner layer cell, a further stage, characterised by a decrease of the current is observed, corresponding to a lowering in the polymerisation rate caused by the depletion of dimer in the solution.

\section{Conclusions}

Several instrumental techniques (EQCM, ellipsometry, monodimensional spectroscopy, etc.) have been currently used in the study of anodic synthesis of conducting polymers. When used separately from one another, they provide for partial information, which is often unsuitable in order to differentiate the phenomena occurring on the electrode surface from those taking place in the solution nearby. Experiments carried out by using bidimensional spectroelectrochemistry give us the possibility of looking 'selectively' at the two distinct main processes, i.e., the deposition of the polymer onto the electrode and the formation of intermediate neutral and oxidised oligomers in the solution. In particular, the bidimensional chronoabsorptometric monitoring of the electropolymerisation of MBTBT allows us to ascertain that the oligomeric species are always formed in the solution before the coverage of the electrode surface starts. The polymer film grows linearly with time, but the concentration of oligomers, after an induction time', reaches a quasi-steady-state during the whole process. An overall reaction scheme can be accordingly proposed for the electropolymerisation.

\section{Acknowledgements}

Support of the DGICYT (PB93-0677) and Junta de Castilla y León (BU11/98) is gratefully acknowledged.

\section{References}

[1] D. Fichou, Handbook of Oligo- and Polythiophenes, Wiley-VCH, Weinheim, 1999.

[2] J. Heinze, Topics in Current Chemistry, vol. 152, Springer, Berlin, 1990.

[3] M.E.G. Lyons, Electroactive Polymer Electrochemistry, Part 1. Fundamentals, Part 2. Methods and Applications, Plenum Press, New York, 1994.

[4] J. Heinze, Topics in Current Chemistry, vol. 152, Springer, Berlin, 1990.
[5] G. Inzelt, in: A.J. Bard (Ed.), Electroanalytical Chemistry, vol. 18, Marcel Dekker, New York, 1994, pp. 89-241.

[6] G. Zotti, G. Schiavon, S. Zecchin, Synth. Met. 72 (1995) 275.

[7] C. Visy, J. Kankare, E. Kriván, Electrochim. Acta 45 (2000) 3851.

[8] R.D. McCullough, Adv. Mater. 10 (2) (1998) 93.

[9] J. Roncali, Chem. Rev. 92 (4) (1992) 711.

[10] J. Roncali, J. Mater. Chem. 9 (1999) 1875.

[11] A. Yassar, P. Valat, V. Wintgens, M. Hmyene, F. Deloffre, G. Horowitz, P. Srivastava, F. Garnier, Synth. Met. 67 (1994) 277.

[12] H. Ding, L. Pigani, R. Seeber, C. Zanardi, J. New. Mat. Electrochem. Systems 3 (2000) 339.

[13] B. Ballarin, F. Costanzo, F. Mori, A. Mucci, L. Pigani, L. Schenetti, R. Seeber, D. Tonelli, C. Zanardi, Electrochim. Acta 46 (2001) 881.

[14] M. Skompska, Electrochim. Acta 44 (1998) 357.

[15] A.R. Hillman, E.F. Mallen, J. Electroanal. Chem. 220 (1987) 351.

[16] K. Meerholz, J. Heinze, Electrochim. Acta 41 (1996) 1839.

[17] J. Heinze, H. John, M. Dietrich, P. Tschuncky, Synth. Met. 119 (2001) 49.

[18] A. Smie, A. Synowczyk, J. Heinze, R. Alle, P. Tschuncky, G. Götz, P. Bäuerle, J. Electroanal. Chem. 452 (1998) 87.

[19] G. Casalbore-Miceli, N. Camaioni, A. Geri, A. Berlin, R. Campesato, Electrochim. Acta 44 (1999) 4781.

[20] G. Casalbore-Miceli, N. Camaioni, A. Geri, M. Cristani, A.M. Fichera, A. Berlin, Synth. Met. 108 (2000) 47.

[21] C. Visy, J. Lukkari, J. Kankare, J. Electroanal. Chem. 319 (1991) 85.

[22] C. Visy, J. Lukkari, J. Kankare, Synth. Met. 55 (1993) 1620.

[23] C. Visy, J. Lukkari, J. Kankare, Macromolecules 26 (1993) 3295.

[24] E. Lankinen, M. Pohjakallio, G. Sundholm, P. Talonen, T. Laitinen, T. Saario, J. Electroanal. Chem. 437 (1997) 167.

[25] G. Louarn, M. Trznadel, J.P. Buisson, J. Laska, A. Pron, M. Lapkowski, S. Lefrant, J. Phys. Chem. 100 (1996) 12532.

[26] A.R. Hillman, E.F. Mallen, J. Electroanal. Chem. 243 (1988) 403.

[27] A.R. Hillman, E.F. Mallen, A. Hamnett, J. Electroanal. Chem. 244 (1988) 353.

[28] M. Olbrich-Stock, J. Posdorfer, R.N. Schindler, J. Electroanal. Chem. 368 (1994) 173.

[29] A.R. Hillman, M.J. Swann, Electrochim. Acta 33 (1988) 1303.

[30] C. Kvarnstrom, H. Neugebauer, S. Blomquist, H.J. Ahonen, J. Kankare, A. Ivaska, Electrochim. Acta 44 (1999) 2739.

[31] E. Lankinen, G. Sundholm, P. Talonen, H. Grano, F. Sundholm, J. Electroanal. Chem. 460 (1999) 176.

[32] S. Garreau, G. Louarn, G. Froyer, M. Lapkowski, O. Chauvet, Electrochim. Acta 46 (2001) 1207.

[33] M. Belletête, L. Mazerolle, N. Desrosiers, M. Leclerc, G. Durocher, Macromolecules 28 (1995) 8587.

[34] C. Visy, J. Kankare, J. Electroanal. Chem. 442 (1998) 175.

[35] H. Ding, Z. Pan, L. Pigani, R. Seeber, C. Zanardi, Electrochim. Acta 46 (2001) 2721.

[36] B. Krische, M. Zagórska, Synth. Met. 33 (1989) 257.

[37] P.A. Christensen, A. Hamnett, A.R. Hillman, J. Electroanal. Chem. 242 (1988) 47.

[38] J. López-Palacios, A. Colina, A. Heras, V. Ruiz, L. Fuente, Anal. Chem. 73 (2001) 2883.

[39] V. Ruiz, A. Colina, A. Heras, J. López-Palacios, R. Seeber, Helv. Chim. Acta 84 (2001) 3628. 